Article

\title{
Emergence of a Multidrug-Resistant Enterobacter hormaechei Clinical Isolate from Egypt Co-Harboring mcr-9 and bla $\mathrm{VIM}-4$
}

\author{
Ahmed M. Soliman ${ }^{1,2}{ }^{\mathbb{D}}$, Fumito Maruyama ${ }^{3}{ }^{-}$, Hoda O. Zarad ${ }^{1}$, Atsushi Ota ${ }^{3}$, \\ Hirofumi Nariya ${ }^{4}$, Toshi Shimamoto ${ }^{4}$ and Tadashi Shimamoto 4 ,* (D) \\ 1 Laboratory of Food Microbiology and Hygiene, Graduate School of Biosphere Science, Hiroshima University, \\ Higashi-Hiroshima 739-8528, Japan; ahmed_soliman@pharm.kfs.edu.eg (A.M.S.); \\ hodaothmanzarad@gmail.com (H.O.Z.) \\ 2 Department of Microbiology and Immunology, Faculty of Pharmacy, Kafrelsheikh University, \\ Kafr El-Sheikh 33516, Egypt \\ 3 Microbial Genomics and Ecology, Office of Academic Research and Industry-Government Collaboration, \\ Hiroshima University, Higashi-Hiroshima 739-8530, Japan; fumito@hiroshima-u.ac.jp (F.M.); \\ leve1997@gmail.com (A.O.) \\ 4 Laboratory of Food Microbiology and Hygiene, Graduate School of Integrated Sciences for Life, Hiroshima \\ University, Higashi-Hiroshima 739-8528, Japan; nariya@hiroshima-u.ac.jp (H.N.); \\ tsima@hiroshima-u.ac.jp (T.S.) \\ * $\quad$ Correspondence: tadashis@hiroshima-u.ac.jp; Tel.: +81-82-424-7897; Fax: +81-82-424-7916
}

Received: 3 April 2020; Accepted: 17 April 2020; Published: 20 April 2020

\begin{abstract}
This study describes the first full genomic sequence of an $m c r-9$ and $b a_{\mathrm{VIM}-4}$-carrying multidrug-resistant Enterobacter hormaechei clinical isolate from Egypt. The strain was isolated in April 2015 from the sputum of a patient in Cairo, Egypt. The $m c r-9$ and $b l a_{\mathrm{VIM}-4}$ genes were identified by PCR screening and DNA sequencing; the isolate was subjected to antimicrobial susceptibility testing, conjugation experiments, and whole genomic sequencing. $m c r-9$ and $b l a_{\mathrm{VIM}-4}$ were carried by an IncHI2 plasmid, pAMS-38a (281,121 bp in size); the plasmid also carried genes conferring resistance against sulfonamides (sul1), quinolones ( $q n r A 1)$, trimethoprim (dfrA1), $\beta$-lactams $\left(b l a_{\mathrm{TEM}-1 \mathrm{~B}}\right)$, aminoglycosides (aac (6')-II, aadA23, aadA2b, and ant (2")-Ia). The strain was susceptible to colistin $(\mathrm{MIC},<0.25 \mu \mathrm{g} / \mathrm{mL}$ ); this could be due to the absence of the $q s e C / q s e B$ regulatory system located downstream of $m c r-9$ in Enterobacterales, which is involved in the induction of colistin-resistance. The genetic context of $m c r-9$ and bla $a_{\mathrm{VIM}-4}$ was identified as IS1-mcr-9-IS903-pcoS- $\Delta p c o E-r c n A$ and

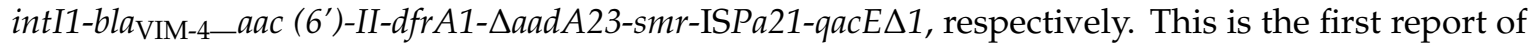
an $m c r-9$ and $b l a_{\mathrm{VIM}-4}$ /IncHI2-carrying multidrug-resistant E. hormaechei clinical isolate from Africa and the Middle East. Plasmids of the IncHI2 group and the two insertion sequences (IS1, and IS903) might be the main vehicles for dissemination of $m c r-9$. Further screening for $m c r-9$ is essential for identifying its incidence and to prevent its dissemination.
\end{abstract}

Keywords: mcr-9; Egypt; VIM-4; IncHI2; WGS; Enterobacter hormaechei

\section{Introduction}

Problems associated with the development and spread of antimicrobial resistance (AMR) in clinical practice are increasing and are currently being viewed as a major threat to public health globally [1,2]. Carbapenem resistance in Enterobacterales is mediated through the expression of a large set of carbapenemase-encoding genes: particularly, Ambler class A $\beta$-lactamases (KPC, FRI, GES, SME), Ambler class B metallo- $\beta$-lactamases (VIM, NDM, IMP, SIM, GIM, SPM), and Ambler class D 
enzymes (OXA-type) [3,4]. Verona integron-mediated metallo- $\beta$-lactamase (VIM) is located as a gene cassette in a class 1 integron with genes for resistance against trimethoprim or aminoglycosides [5]. The $b l a_{\mathrm{VIM}-4}$ gene encodes resistance against all $\beta$-lactams except aztreonam and was previously reported in Pseudomonas aeruginosa, Enterobacter cloacae, or other Enterobacterales from Egypt, Kuwait, and United Arab Emirates [5-8]. Recently, we reported bla $a_{\mathrm{VIM}-2}$ and bla $a_{\mathrm{VIM}-24}$ from clinical P. aeruginosa strains from Egypt [5].

Colistin is one of the last-resort antibiotics for treating infections caused by carbapenem resistant Gram-negative bacteria. The activity and efficacy of colistin has been challenged by the global spread of the self-transmissible mobilized colistin-resistance genes ( $m c r$ ). In 2016, the first $m c r-1$ gene was reported from Escherichia coli and Klebsiella pneumoniae isolated from patients, food, and animals in China [9]. MCR-1 acts by modifying the lipid A part of the lipopolysaccharide in Gram-negative bacteria by adding phosphoethanolamine, which reduces the binding affinity to colistin [9]. As of 7 January 2020, 10 variants of the mor genes are available in the Bacterial Antimicrobial Resistance Reference Gene Database (https://www.ncbi.nlm.nih.gov/pathogens/isolates\#/refgene/); however, only $m c r-1$ has been previously reported from Egypt [10], and little is yet known about the prevalence of other $m c r$ variants in Egypt.

Here, for the first time, we describe the complete genomic sequence of an E. hormaechei clinical isolate carrying the $m c r-9$ and $b l a_{\mathrm{VIM}-4}$ genes on an IncHI2 plasmid from Africa and the Middle East generated using the Illumina MiniSeq and Oxford Nanopore sequencing platforms.

\section{Materials and Methods}

\subsection{Bacterial Isolation and Identification}

The E. hormaechei strain AMS-38 was isolated from the sputum swab of a patient in Cairo, Egypt in April 2015. The strain was identified by $16 \mathrm{~S}$ ribosomal RNA gene sequencing using primers $27 \mathrm{~F}$ and 1492R [5]. PCR and DNA sequencing were conducted to identify extended-spectrum $\beta$-lactamases, carbapenemase-encoding genes, $16 \mathrm{~S}$ rRNA methylases, plasmid-mediated quinolone-resistance genes, and plasmid-mediated colistin-resistance genes $(m c r-1-m c r-8)$ as previously described [5]. Additionally, $m c r-9$ was detected by primers $m c r-9$-forward: $5^{\prime}$-CGGTACCGCTACCGCAATAT- $3^{\prime}$ and $m c r-9$-reverse: 5'-ATAACAGCGAGACACCGGTT-3' [11].

\subsection{Antimicrobial Susceptibility Testing (AST)}

A standardized broth microdilution (BMD) technique was used to determine the minimum inhibitory concentration (MIC) of various antimicrobial agents according to the standards and interpretive criteria described by the Clinical and Laboratory Standards Institute (CLSI, document M100-S24) and European Committee on Antimicrobial Susceptibility Testing (EU-CAST) (for colistin and tigecycline breakpoints) (http://www.eucast.org). For all experiments, the purified powder of each antibiotic was diluted following CLSI recommendations. E. coli ATCC 25922 was used as a control.

\subsection{Filter-Mating Conjugation}

A Conjugation experiment was performed using the AMS-38 strain and the azide resistant E. coli J53 strain as the donor and recipient, respectively. Due to the thermo-susceptibility of IncHI2 plasmids (i.e., conjugation is impaired at $37{ }^{\circ} \mathrm{C}$ ), we performed this experiment at $25-30{ }^{\circ} \mathrm{C}$ [12]. The transconjugants were selected on LB agar plates containing $100 \mu \mathrm{g} / \mathrm{mL}$ ampicillin and $100 \mu \mathrm{g} / \mathrm{mL}$ sodium azide. Colony-direct PCR was performed using VIM-F and VIM-R [5] and mcr-9-forward and $m c r-9$-reverse [11] primers to confirm the transfer of the plasmid carrying $m c r-9$ and $b l a_{\mathrm{VIM}-4}$.

\subsection{Whole Genome Sequencing (WGS) and Analysis}

An overnight bacterial culture of the AMS-38 strain was used to extract the total genomic DNA (gDNA) using a standard proteinase $\mathrm{K}$ method. The quality of the isolated gDNA was assessed 
using a Microdrop (Thermo Scientific ${ }^{\mathrm{TM}}$, Multiskan ${ }^{\mathrm{TM}}$ Sky Microplate Spectrophotometer, Waltham, MA, USA), Bioanalyzer (Agilent Technologies, Santa Clara, CA, USA), and a dsDNA fluorescent dye method using DeNovix (Wilmington, DE, USA). The sequencing library was constructed using a Nextera DNA Flex Library Prep Kit (Illumina, San Diego, CA, USA.) for Illumina MiniSeq. The DNA library for Oxford Nanopore sequencing was prepared using $400 \mathrm{ng}$ of gDNA according to the Rapid Barcoding Sequencing kit (SQK-RBK004) (Oxford Nanopore Technologies, Oxford, UK). The sequencing libraries were purified using a Promega Size-Selective Purification System (Promega, Madison, WI, USA), and $11 \mu \mathrm{L}$ of the sequencing library was loaded onto a FLO-MIN106 flow cell and sequenced with the GridION device (Oxford Nanopore Technologies) for $48 \mathrm{~h}$. Hybrid assembly of both Illumina and Nanopore reads was performed using Flye v2.6 (https://github.com/fenderglass/Flye). Additionally, Pilon-v1.23 [13] was used to improve the genome assembly. The assembly and annotation of pAMS-38a were performed using DFAST [14]. The identification of AMS-38 was done with JSpeciesWS (http://jspecies.ribohost.com/jspeciesws/\#analyse) using Tetra Correlation Search (TCS) against reference database Genome DB and the pairwise comparison using Average Nucleotide Identity calculation based on BLAST+ (ANIb). Antimicrobial resistance genes were identified by ResFinder-3.2 available at Center for Genomic Epidemiology (https://cge.cbs.dtu.dk/services/ResFinder/). The plasmid Inc groups, pMLST and multilocus sequence typing (MLST) were identified by PlasmidFinder (https://cge.cbs.dtu.dk/services/PlasmidFinder/), (https://cge.cbs.dtu.dk/services/pMLST/) and MLST 2.0 software (https://cge.cbs.dtu.dk/services/MLST/), respectively. The plasmids that were highly similar to pAMS-38a were detected by a Mash (v2.1.1) [15] distance search against the PLSDB v. 2019_10_07 [16]. The BRIG tool was used to perform a circular comparison between the complete sequence of pAMS-38 and the highly similar plasmids detected by a Mash distance search [17]. EasyFig tool was used to visualize and compare the genetic environment of $m c r-9$ in its harboring plasmids (http://mjsull.github.io/Easyfig/).

\subsection{Nucleotide Sequence Accession Numbers}

The complete genome sequence of AMS-38 and the plasmids pAMS-38a, pAMS-38b, pAMS-38c, pAMS-38d were submitted to DDBJ/ENA/GenBank under BioProject ID: PRJNA616711.

\section{Results and Discussion}

\subsection{AST of E. hormaechei AMS-38, and Conjugation of mcr-9 and bla ${ }_{V I M-4}$-Carrying Plasmid}

The sequencing of the $16 \mathrm{~S}$ ribosomal RNA gene identified a strain belonging to the E. cloacae complex. BMD showed that the strain AMS-38 was resistant to aztreonam (MIC, $32 \mu \mathrm{g} / \mathrm{mL}$ ), ceftazidime (MIC, $>256 \mu \mathrm{g} / \mathrm{mL}$ ), ceftriaxone (MIC, $>256 \mu \mathrm{g} / \mathrm{mL}$ ), meropenem (MIC, $64 \mu \mathrm{g} / \mathrm{mL}$ ), ertapenem (MIC, $>64 \mu \mathrm{g} / \mathrm{mL}$ ), kanamycin (MIC, $64 \mu \mathrm{g} / \mathrm{mL}$ ), ciprofloxacin (MIC, $2 \mu \mathrm{g} / \mathrm{mL}$ ), fosfomycin (MIC, $128 \mu \mathrm{g} / \mathrm{mL}$ ), tetracycline (MIC, $16 \mu \mathrm{g} / \mathrm{mL}$ ), and tigecycline (MIC, $4 \mu \mathrm{g} / \mathrm{mL}$ ). The strain showed intermediate resistance to chloramphenicol (MIC, $16 \mu \mathrm{g} / \mathrm{mL}$ ) and sensitivity to colistin (MIC, $<0.25 \mu \mathrm{g} / \mathrm{mL}$ ). PCR screening and DNA sequencing identified the presence of the metallo- $\beta$-lactamase genes, bla $a_{\mathrm{VIM}-4}, b l a_{\mathrm{TEM}-1}$, AmpC $\beta$-lactamase, the newly described inducible colistin-resistance gene $m c r-9$, the fosfomycin glutathione-S-transferase gene fos $A$, and the plasmid-mediated quinolone-resistance gene qnrA1. The results showed that the $m c r-9$ and $b l a_{\mathrm{VIM}-4}$-carrying plasmid was successfully transferred to $E$. coli J53. The intact conjugative transfer locus in pAMS-38a was similar to that locus in plasmid pME-1a (accession no. CP041734), and found in two distinct regions, Tra1 and Tra2 illustrating the potential for horizontal gene transfer to other Gram-negative bacteria.

\subsection{Characterization of the Genome of E. hormaechei AMS-38}

By combining the short reads obtained from Illumina MiniSeq and the long reads obtained from Oxford Nanopore sequencing, we obtained assemblies with high-quality and sufficient for closing the genome and the plasmids contained in the strain. The chromosome of the AMS-38 strain was 
4,914,941 bp in size with an average $\mathrm{G}+\mathrm{C}$ content of $54.9 \%$. Analyzing the average nucleotide identity (ANI) of the AMS-38 genome showed that the strain belonged to E. hormaechei subsp. steigerwaltii group $(99.69 \%$ identity (ID) with $97.71 \%$ query coverage(QC) to E. hormaechei subsp. steigerwaltii (GCA 001011725) GN02001 followed by Enterobacter sp. MGH86 (99.62\% ID with $95.21 \%$ QC), followed by E. hormaechei subsp. steigerwaltii 44524 (98.36\% ID with $86.91 \%$ QC), followed by E. hormaechei subsp. steigerwaltii 44517 (98.36\% ID with 86.92 QC)). Analysis of MLST of the strain AMS-38 showed that it belonged to ST133 (allelic profile 11-4-4-13-39-4-9).

\subsection{Plasmids and Resistome Analysis of the Strain E. hormaechei AMS-38}

The isolate contained four plasmids (pAMS-38a, pAMS-38b, pAMS-38c, pAMS-38d) ranging in size from 2,511 bp to $281,121 \mathrm{bp}$ (Table 1). ResFinder detected that the intrinsic bla $a_{\mathrm{ACT}-7}$ and fos $A$ genes were located on the chromosome. Additionally, plasmid pAMS-38a contained 10 antimicrobial resistance genes encoding resistance against colistin ( $(m c r-9)$, sulphonamide (sul1), quinolones (qnrA1), trimethoprim (dfrA1), $\beta$-lactam (bla $a_{\mathrm{TEM}-1 \mathrm{~B}}$, and bla $\left.a_{\mathrm{VIM}-4}\right)$, and aminoglycosides (aac (6')-II, aadA23, $a a d A 2 b$, and ant $(2 ")-I a)$. The remaining three plasmids did not carry any resistance genes.

Table 1. Features of chromosome and the plasmids identified in E. hormaechei AMS-38 from Egypt.

\begin{tabular}{|c|c|c|c|c|c|c|}
\hline Sample & Size (bp) & GC\% & $\begin{array}{l}\text { No. of } \\
\text { CDSs }\end{array}$ & $\begin{array}{c}\text { MLST or } \\
\text { pMLST }\end{array}$ & Inc Type * & AMR Gene \\
\hline Chromosome & $4,914,941$ & 54.9 & 6279 & ST133 & ND & fos $A, b l a_{\mathrm{ACT}-7}$ \\
\hline pAMS-38a & 281,121 & 46.8 & 361 & ST1 & IncHI2 & $\begin{array}{l}\text { mcr-9, sul1, qnrA1, dfrA1, } \\
\text { bla } \\
\text { aaM-1B, bla }{ }_{\mathrm{VIM}-4}, \text { aac }\left(6^{\prime}\right)-I l, \\
\text { adA23, aadA2b, ant }\left(2^{\prime \prime}\right)-I a\end{array}$ \\
\hline pAMS-38b & 109,015 & 50.8 & 144 & ND & IncFIB(pHCM2) & ND \\
\hline pAMS-38c & 9525 & 51.7 & 15 & ND & ND & ND \\
\hline pAMS-38d & 2511 & 51.8 & 2 & ND & ND & ND \\
\hline
\end{tabular}

Plasmid pAMS-38a is an IncHI2 with Double Locus Sequence Type DLST1 of 281,121 bp in size encoding 361 predicted genes with an average $\mathrm{G}+\mathrm{C}$ content of $46.8 \%$ (Figure 1 and Table 1). Plasmids of IncHI type are large in size ( $>200 \mathrm{~kb}$ ) [12]. These plasmids have broad host range and have been commonly detected from several Enterobacterales species of clinical and environmental origin, such as Salmonella enterica serovars Typhi and Paratyphi A, E. coli, K. pneumoniae, and E. cloacae [12]. IncHI2 plasmids are characterized by carrying genes encoding heavy metal and antimicrobial resistance [12].

A Mash distance search against the PLSDB using the whole pAMS-38a sequence identified that it has high similarity to other IncHI2 plasmids (Figure 1 and Table 2), e.g., pC45-VIM4 (accession no. LT991958), pME-1a (accession no. CP041734) [18], pCTXM9_020038 (accession no. CP031724), pMRVIM0813 (accession no. KP975077), pKPC-272 (accession no. CP008825), p707804-NDM (accession no. MH909331), pT5282-mphA (accession no. KY270852), and pN1863-HI2 (accession no. MF344583). Alarmingly, all these plasmids carried the $m c r-9$ gene with or without carbapenemase-encoding genes (i.e., $b l a_{\mathrm{VIM}}, b l a_{\mathrm{KPC}}$, and $b l a_{\mathrm{NDM}}$ ) between 2012 to 2018, highlighting the early silent emergence and dissemination of $m c r-9$. A recent study conducted in the USA identified a similar $m c r-9$ and bla $a_{\mathrm{VIM}-4} / \mathrm{IncHI} 2$ plasmid pME-1a (accession no. CP041734) from an E. hormaechei strain isolated from a pediatric patient with a recent history of travel to Egypt [18]. Therefore, our study raises the concern of implementing antimicrobial surveillance plans and infection control policies for $m c r-9$ to identify its incidence and to prevent its dissemination. 


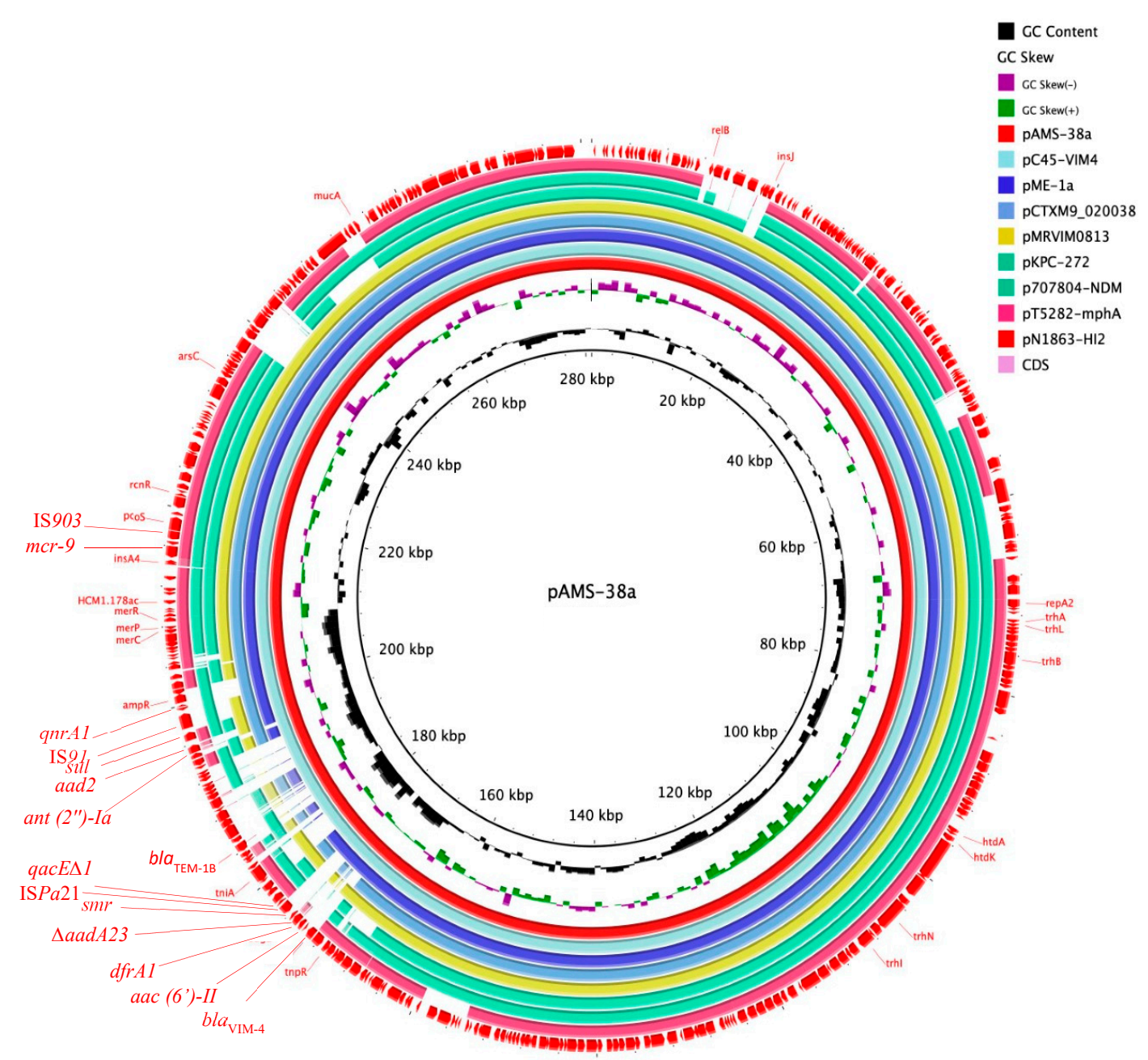

Figure 1. Plasmid structure of $m c r-9 / \mathrm{IncHI} 2$ plasmids. The whole sequence of pAMS-38a was used as the reference. Genes and open reading frames (ORFs) are shown as arrows with their transcriptional orientations indicated by arrowheads. This figure was generated using the BRIG tool [17], and the plasmids were included in the following order: pAMS-38a (identified in this study), pC45-VIM4 (LT991958), pME-1a (CP041734), pCTXM9_020038 (CP031724), pMRVIM0813 (KP975077), pKPC-272 (CP008825), p707804-NDM (MH909331), pT5282-mphA (KY270852), and pN1863-HI2 (MF344583).

Table 2. Features of $m c r-9 / I n c H I 2$ plasmids similar to pAMS-38a.

\begin{tabular}{ccccccc}
\hline Plasmid & $\begin{array}{c}\text { Distance To } \\
\text { pAMS-38a }\end{array}$ & Bacterial Species & $\begin{array}{c}\text { Isolation } \\
\text { Year }\end{array}$ & Country & Accession No. \\
\hline 1 & pC45-VIM4 & 0.0022 & E. cloacae complex & 2014 & France & LT991958 \\
\hline 2 & pME-1a & 0.0026 & $\begin{array}{c}\text { E. hormaechei } \\
\text { subsp. steigerwaltii }\end{array}$ & 2018 & USA & CP041734 \\
\hline 3 & pCTXM9_020038 & 0.0048 & E. hormaechei & 2016 & China & CP031724 \\
\hline 4 & pMRVIM0813 & 0.0049 & E. cloacae & 2015 & USA & KP975077 \\
\hline 5 & pKPC-272 & 0.0079 & E. cloacae & 2012 & USA & NZ_CP008825.1 \\
\hline 6 & p707804-NDM & 0.0080 & $\begin{array}{c}\text { Leclercia } \\
\text { adecarboxylata }\end{array}$ & ND & China & MH909331.1 \\
\hline 7 & pT5282-mphA & 0.0108 & E. cloacae & 2012 & China & KY270852 \\
\hline 8 & pN1863-HI2 & 0.0120 & E. cloacae & ND & China & MF344583 \\
\hline
\end{tabular}

* as detected by a Mash distance search against the PLSDB using the whole pAMS-38a sequence; ${ }^{* *}$ ND: not determined. 
Analysis of the genetic environment of $m c r-9$ revealed that it was surrounded upstream by IS903 and downstream by IS1. Additionally, these two insertion sequences might be involved in the mobilization of $m c r-9$. This genetic environment has been reported previously from several IncHI2 plasmids (Figure 2) e.g., pME-1a (accession no. CP041734) [18], pCTXM9_020038 (accession no. CP031724), and pMRVIM0813 (accession no. KP975077). A different genetic organization, qseB-qseC-wbuC-mcr-9-IS903, has been detected from other IncHI2 plasmids e.g., pN1863-HI2 (accession no. MF344583), pT5282-mphA (accession no. KY270852), pMRVIM0813 (accession no. KP975077), and the sequenced plasmid contig from E. coli strain E68 from France [11]. In that study, Kieffer et al. showed that $m c r-9$ is an acquired colistin-resistance gene induced by the action of the downstream two-component regulatory system encoded by qseB/qseC using subinhibitory concentrations of colistin [11]. Another study was conducted on a clinical E. hormaechei isolate from China with the coproduction of MCR-9 and NDM-1 [19]. The investigators identified mcr-9 to be located on an IncHI2 plasmid with the downstream genes $w b u C$ (for a cupin fold metalloprotein) and the $q s e B / q s e C$ leading to colistin-resistance with an MIC of $16 \mu \mathrm{g} / \mathrm{mL}$ [19]. In our study, the sensitivity of the strain AMS-38 to colistin (MIC, $<0.25 \mu \mathrm{g} / \mathrm{mL}$ ) was probably due to the absence of $q s e B / q s e C$. It has been noted that mcr-9 might have originated from Buttiaxella spp. due to the presence of a gene homologous to wbuC downstream of the chromosomal mcr-9-like gene in this genus [11,19]. Of note, $m c r-9$ was first identified, during a routine in silico analysis, in a multidrug- resistant colistin-sensitive S. enterica serovar Typhimurium clinical isolate from the USA in 2010 [20]. Recently, mcr-9 was detected among SHV-12-producing Enterobacterales from Swedish horses with a link to IncHI2 and IncHI2A plasmids [21]. Additionally, an extensively drug-resistant bla $a_{\mathrm{NDM}-1}$-carrying Klebsiella quasipneumoniae was detected to harbor $m c r-9$ on an IncHI2 plasmid for the first time in Latin America [22]. A novel mobile colistin resistance gene, $m c r-10$, was recently reported from a clinical Enterobacter roggenkampii isolate in China [23]. MCR-10 has $82.93 \%$ amino acids identity to MCR-9 [23]. Gene $m c r-10$ was detected to be uninducible and increases the colistin MIC by 4 folds when cloned into a colistin-susceptible E. roggenkampii strain [23].

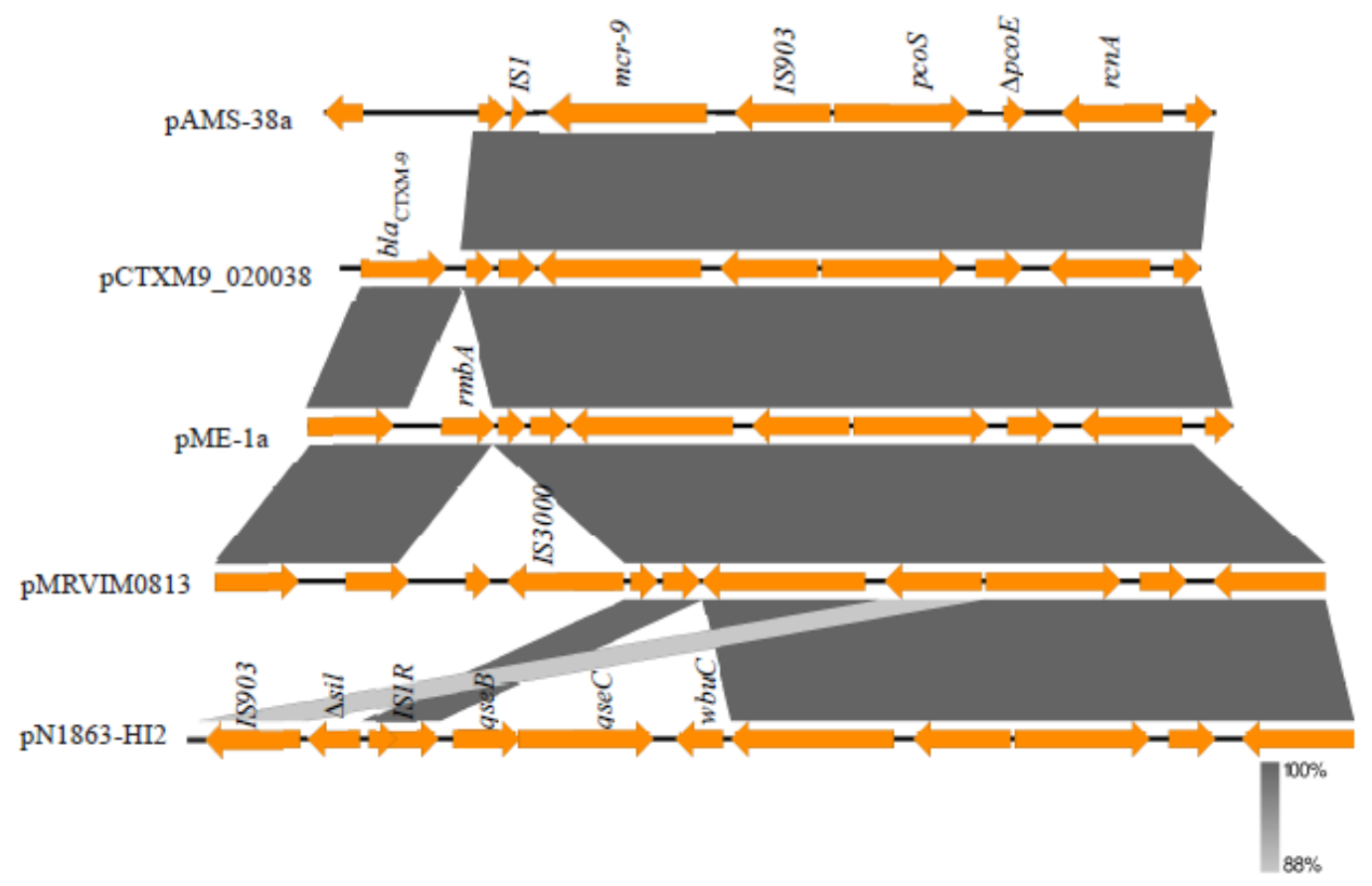

Figure 2. Comparison of the genetic environment of $m c r-9$ gene in pAMS-38a and other closely related IncHI2 plasmids. The figure was drawn using the EasyFig tool. Two different genetic environments have been detected. IS1-mcr-9-IS903 was detected from pAMS-38a (identified in this study), pME-1a (CP041734), pCTXM9_020038 (CP031724), pMRVIM0813 (KP975077), and qseB-qseC-wbuC-mcr-9-IS903 was detected from $\mathrm{pN} 1863-\mathrm{HI} 2$ (MF344583). 
Based on the available literature, the frequency of detecting $m c r-9$-carrying Enterobacterales with susceptibility to polymyxins is high [18,20-22]. Additionally, we speculate a silent dissemination of $m c r-9$ since its possession confers a decreased susceptibility, not resistance, to colistin as detected in several Enterobacterales [18,20-22]. On the other hand, only two studies detected mcr-9 conferring clinical resistance to colistin [11,19]. Moreover, the expression of $m c r-9$ was shown to be inducible due to the presence of the downstream $q s e C / q s e B$ regulatory system [11]. Furthermore, the lack of these two genes downstream of the mcr-9-like gene in its original progenitor, B. gaviniae, suggests that their acquisition likely has been through an independent mobilization event with respect to the acquisition of $m c r-9$ [11]. Similarly, that event might happen in $m c r-9$-carrying and colistin susceptible strains leading to the induction and colistin resistance. Until now, the clinical impact of $m c r-9$, in the colistin-susceptible strains lacking qseC/qseB system, is unknown.

The class B1 metallo- $\beta$-lactamase $b l a_{\mathrm{VIM}-4}$ was located on an In 416 class 1 integron. A BLASTn search using the whole bla $a_{\mathrm{VIM}-4}$ integron sequence of pAMS-38a identified identical integrons from pC45-VIM4 (accession no. LT991958), pME-1a [18], pC309-VIM4 (accession number LT991955.1) (100\% query coverage and $99.97 \%$ sequence identity). bla $a_{\mathrm{VIM}-4}$ has been previously reported from E. cloacae and P. aeruginosa from Egypt, Kuwait, and United Arab Emirates [6-8,24]. The integron structure

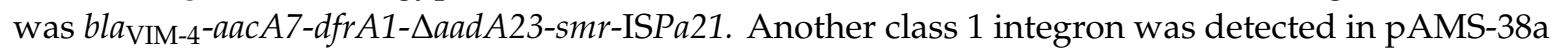
with the following genetic structure: intI1-ant (2")-Ia-aad2b-sul1-IS91-qnrA1. A BLASTn analysis identified a similar integron from pCTXM9_020038 (accession no. CP031724; 100\% query coverage and 99.88\% sequence identity), and pC45-VIM4 (accession no. LT991958; 100\% query coverage and 99.87\% sequence identity).

\section{Conclusions}

In conclusion, we reported the first complete genomic sequence of an mcr-9- and bla $a_{\mathrm{VIM}-4}$-co- $^{-\mathrm{co}}$ harboring E. hormaechei strain from Africa and the Middle East. Comparative genomics was used to assess the genetic environment of the $m c r-9$ gene. Plasmids of the IncHI2 group and the two insertion sequences (IS1, and IS903) might be the main vehicles for dissemination of $m c r-9$. Screening for $m c r-9$ and other $m c r$ genes is essential in Egypt to determine its prevalence and to prevent its spread.

Author Contributions: Conceptualization, A.M.S. and T.S. (Tadashi Shimamoto); Data curation, A.M.S., F.M., H.O.Z., A.O. and T.S. (Toshi Shimamoto); Formal analysis, A.M.S. and F.M.; Funding acquisition, T.S. (Tadashi Shimamoto); Investigation, A.M.S. and H.N.; Methodology, A.M.S., F.M., H.O.Z., A.O., H.N. and T.S. (Toshi Shimamoto); Project administration, T.S. (Tadashi Shimamoto); Software, A.M.S. and F.M.; Validation, A.M.S.; Writing-original draft, A.M.S.; Writing-review \& editing, A.M.S., F.M. and T.S. (Tadashi Shimamoto). All authors have read and agreed to the published version of the manuscript.

Acknowledgments: A.M.S. was supported by a fellowship from the Ministry of Education, Culture, Sports, Science, and Technology of Japan (Fellowship no. 153532). F.M. was supported by the Japan Agency for Medical Research (AMED; project number 19fk0108043h8003).

Conflicts of Interest: The authors declare no conflict of interest.

\section{References}

1. World Health Organization (WHO). Antimicrobial Resistance: Global Report on Surveillance 2014; WHO Press: Geneva, Switzerland, 2014.

2. Centers for Disease Control and Prevention (CDC). Antibiotic resistance threats in the United States, 2013. Available online: http://www.cdc.gov/drugresistance/threat-report-2013/pdf/arthreats-2013-508.pdf (accessed on 24 March 2020).

3. Hsu, L.Y.; Apisarnthanarak, A.; Khan, E.; Suwantarat, N.; Ghafur, A.; Tambyah, P.A. Carbapenem-resistant Acinetobacter baumannii and Enterobacteriaceae in South and Southeast Asia. Clin. Microbiol. Rev. 2016, 30, 1-22. [CrossRef]

4. Meunier, D.; Findlay, J.; Doumith, M.; Godoy, D.; Perry, C.; Pike, R.; Gronthoud, F.; Shryane, T.; Poirel, L.; Welfare, W.; et al. FRI-2 carbapenemase-producing Enterobacter cloacae complex in the UK. J. Antimicrob. Chemother. 2017, 72, 2478-2482. [CrossRef] [PubMed] 
5. Soliman, A.M.; Zarad, H.O.; Nariya, H.; Shimamoto, T.; Shimamoto, T. Genetic analysis of carbapenemaseproducing Gram-negative bacteria isolated from a university teaching hospital in Egypt. Infect. Genet. Evol. 2020, 77, 104065. [CrossRef] [PubMed]

6. Hashem, H.; Hanora, A.; Abdalla, S.; Shaeky, A.; Saad, A. Dissemination of metallo- $\beta$-lactamase in Pseudomonas aeruginosa isolates in Egypt: Mutation in bla $a_{\mathrm{VIM}-4}$. APMIS 2017, 125, 499-505. [CrossRef] [PubMed]

7. Sonnevend, A.; Ghazawi, A.; Yahfoufi, N.; Al-Baloushi, A.; Hashmey, R.; Mathew, M.; Tariq, W.Z.; Pal, T. VIM-4 carbapenemase-producing Enterobacter cloacae in the United Arab Emirates. Clin. Microb. Infect. 2012, 18, E494-E496. [CrossRef]

8. Jamal, W.; Rotimi, V.O.; Albert, M.J.; Khodakhast, F.; Nordmann, P.; Poirel, L. High prevalence of VIM-4 and NDM-1 metallo- $\beta$-lactamase among carbapenem-resistant Enterobacteriaceae. J. Med. Microbiol. 2013, 62, 1239-1244. [CrossRef]

9. Liu, Y.Y.; Wang, Y.; Walsh, T.R.; Yi, L.X.; Zhang, R.; Spencer, J.; Doi, Y.; Tian, G.; Dong, B.; Huang, X.; et al. Emergence of plasmid-mediated colistin resistance mechanism MCR-1 in animals and human beings in China: A microbiological and molecular biological study. Lancet Infect. Dis. 2016, 16, 161-168. [CrossRef]

10. Elnahriry, S.S.; Khalifa, H.O.; Soliman, A.M.; Ahmed, A.S.; Hussein, A.M.; Shimamoto, T.; Shimamoto, T. Emergence of plasmid-mediated colistin resistance gene $\mathrm{mcr}-1$ in a clinical Escherichia coli isolate from Egypt. Antimicrob. Agents Chemother. 2016, 60, 3249-3250. [CrossRef]

11. Kieffer, N.; Royer, G.; Decousser, J.W.; Bourrel, A.S.; Palmieri, M.; De La Rosa, J.M.; Jacquier, H.; Denamur, E.; Nordmann, P.; Poirel, L. mcr-9, an inducible gene encoding an acquired phosphoethanolamine transferase in Escherichia coli, and its origin. Antimicrob. Agents Chemother. 2019, AAC-00965. [CrossRef]

12. Liang, Q.; Yin, Z.; Zhao, Y.; Liang, L.; Feng, J.; Zhan, Z.; Wang, H.; Song, Y.; Tong, Y.; Wu, W.; et al. Sequencing and comparative genomics analysis of the IncHI2 plasmids pT5282-mphA and p112298-catA and the IncHI5 plasmid pYNKP001-dfrA. Int. J. Antimicrob. Agents 2017, 49, 709-718. [CrossRef]

13. Walker, B.J.; Abeel, T.; Shea, T.; Priest, M.; Abouelliel, A.; Sakthikumar, S.; Cuomo, C.A.; Zeng, Q.; Wortman, J.; Young, S.K.; et al. Pilon: An integrated tool for comprehensive microbial variant detection and genome assembly improvement. PLoS ONE 2014, 9, e112963. [CrossRef] [PubMed]

14. Tanizawa, Y.; Fujisawa, T.; Nakamura, Y. DFAST: A flexible prokaryotic genome annotation pipeline for faster genome publication. Bioinformatics 2018, 34, 1037-1039. [CrossRef] [PubMed]

15. Ondov, B.D.; Treangen, T.J.; Melsted, P.; Mallonee, A.B.; Bergman, N.H.; Koren, S.; Phillippy, A.M. Mash: Fast genome and metagenome distance estimation using MinHash. Genome Biol. 2016, 17, 132. [CrossRef] [PubMed]

16. Galata, V.; Fehlmann, T.; Backes, C.; Keller, A. PLSDB: A resource of complete bacterial plasmids. Nucleic Acids Res. 2019, 47, D195-D202. [CrossRef]

17. Alikhan, N.F.; Petty, N.K.; Zakour, N.L.; Beatson, S.A. BLAST Ring Image Generator (BRIG): Simple prokaryote genome comparisons. BMC Genomics 2011, 12, 402. [CrossRef]

18. Chavda, K.D.; Westblade, L.F.; Satlin, M.J.; Hemmert, A.C.; Castanheira, M.; Jenkins, S.G.; Chen, L.; Kreiswirth, B.N. First report of bla $a_{\mathrm{VIM}-4}$ - and $m c r-9$-coharboring Enterobacter species isolated from a pediatric patient. mSphere 2019, 4, e00629-19. [CrossRef]

19. Yuan, Y.; Li, Y.; Wang, G.; Li, C.; Xiang, L.; She, J.; Yang, Y.; Zhong, F.; Zhang, L. Coproduction of MCR-9 and NDM-1 by colistin-resistant Enterobacter hormaechei isolated from bloodstream infection. Infect. Drug Resist. 2019, 12, 2979. [CrossRef]

20. Carroll, L.M.; Gaballa, A.; Guldimann, C.; Sullivan, G.; Henderson, L.O.; Wiedmann, M. Identification of novel mobilized colistin resistance gene $\mathrm{mcr}-9$ in a multidrug-resistant, colistin-susceptible Salmonella enterica serotype Typhimurium isolate. mBio 2019, 10, e00853-19. [CrossRef]

21. Börjesson, S.; Greko, C.; Myrenås, M.; Landén, A.; Nilsson, O.; Pedersen, K. A link between the newly described colistin resistance gene $m c r-9$ and clinical Enterobacteriaceae isolates carrying bla $_{\mathrm{SHV}-12}$ from horses in Sweden. J. Glob. Antimicrob. Resist. 2020, 20, 285-289. [CrossRef]

22. Faccone, D.; Martino, F.; Albornoz, E.; Gomez, S.; Corso, A.; Petroni, A. Plasmid carrying mcr-9 from an extensively drug-resistant NDM-1-producing Klebsiella quasipneumoniae subsp. quasipneumoniae clinical isolate. Infect. Genet. Evol. 2020, 81, 104273. [CrossRef] 
23. Wang, C.; Feng, Y.; Liu, L.; Wei, L.; Kang, M.; Zong, Z. Identification of novel mobile colistin resistance gene mcr-10. Emerg. Microbes Infect. 2020, 9, 508-516. [CrossRef] [PubMed]

24. Dimude, J.U.; Amyes, S.G.B. Molecular characterisation and diversity in Enterobacter cloacae from Edinburgh and Egypt carrying bla $a_{\mathrm{CTX}-\mathrm{M}-14}$ and bla $a_{\mathrm{VIM}-4} \beta$-lactamase genes. Int. J. Antimicrob. Agents 2013, 41, 574-577. [CrossRef] [PubMed]

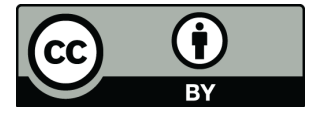

(C) 2020 by the authors. Licensee MDPI, Basel, Switzerland. This article is an open access article distributed under the terms and conditions of the Creative Commons Attribution (CC BY) license (http://creativecommons.org/licenses/by/4.0/). 